\title{
Vitamina D en enfermedades respiratorias: desde la influenza hasta el COVID-19
}

\author{
Alonso Cortina-Gutiérrez¹, Víctor Leal-Martínez², Emilio Abuabara-Franco², Nehomar Pájaro-Galvis ${ }^{3 *}$, \\ María Raad-Sarabia ${ }^{3}$, Jorge Rico-Fontalvo ${ }^{4}$, Rodrigo Daza-Arnedo e Iván de Jesús-Baños ${ }^{6}$
}

${ }^{1}$ Servicio de Reumatología, Universidad del Sinú, Córdoba; ${ }^{2}$ Universidad del Sinú, Córdoba; ${ }^{3}$ Servicio de Medicina Interna, Facultad de Ciencias de la Salud, Universidad del Sinú, Córdoba; ${ }^{4}$ Servicio de Nefrología, Asociación Colombiana de Nefrología, Medellín; ${ }^{5}$ Servicio de Nefrología, Asociación Colombiana de Nefrología, Cartagena; ${ }^{6}$ Servicio de Neumología, Centro Pulmonar Integral, Cartagena. Colombia

\section{Resumen}

La vitamina $D$ activa (1,25-dihidroxicolecalciferol) es una hormona esteroidea que posee múltiples funciones, entre ellas el metabolismo del calcio-fósforo y la mineralización ósea; empero, también tiene un papel fundamental en el funcionamiento del sistema inmunológico y es una herramienta imprescindible en la respuesta contra las infecciones bacterianas (incluidas las micobacterias), micóticas y virales. Se ha demostrado una sólida relación entre la hipovitaminosis $D$ y el desarrollo de infecciones de las vías respiratorias, como neumonía, tuberculosis, infección por virus de la influenza, y aun la pandemia actual por COVID-19. Allí radica la importancia de identificar en fase temprana a los pacientes con deficiencia de vitamina $D$; el objetivo es corregir sus concentraciones y prevenir procesos infecciosos que eleven la morbimortalidad.

Palabras clave: Deficiencia de vitamina D. Infecciones por coronavirus. Sars-CoV. Sistema inmunológico. Mycobacterium tuberculosis. Neumonía.

\section{Vitamin D in respiratory diseases: from the influenza to the COVID-19}

\section{Abstract}

Active vitamin D (1,25-dihydroxycholecalciferol) is a steroid hormone that has multiple functions, including: calcium-phosphorus metabolism and bone mineralization; but, it also has a fundamental role in the functioning of the immune system, being an essential tool in the response against bacterial infections (including mycobacteria), fungi and viruses. A strong association between hypovitaminosis $D$ and the development of respiratory tract infections such as pneumonia, tuberculosis, influenza virus infection, up to the current pandemic COVID-19 has been demonstrated. Hence the importance of identifying patients with vitamin $D$ deficiency early, in order to correct their levels and prevent infectious processes that increase morbidity and mortality.

Key words: Vitamin D deficiency. Coronavirus infections. Sars-CoV. Immune system. Mycobacterium tuberculosis. Pneumonia.

\section{Correspondencia:}

*Nehomar Pájaro-Galvis

E-mail: neho94@ hotmail.com licencia CC BY-NC-ND (http://creativecommons.org/licenses/by-nc-nd/4.0/).
Fecha de recepción: 15-07-2020

Fecha de aceptación: 22-10-2020

DOI: 10.24875 /NEFRO.20000033
Disponible en internet: 17-12-2020

Nefro Latinoam. 2020;17:117-124 www.nefrologialatinoamericana.com 


\section{Introducción}

La vitamina $\mathrm{D}$, una hormona esteroidea producida de forma endógena (también puede adquirirse de la dieta), ha demostrado su papel en la regulación del metabolismo del fosforo y el calcio, que es su función más conocida. Sin embargo, en la última década numerosos estudios han evaluado su función en la activación del sistema inmunitario. Múltiples protocolos han demostrado que la vitamina D mejora la inmunidad innata a través de la inducción de péptidos antimicrobianos, como la catelicidina humana LL-37. Por otra parte, la vitamina $D$ también mejora la inmunidad innata de tipo celular al reducir la tormenta de citocinas proinflamatorias inducida por el sistema inmunitario innato como reacción a infecciones virales (incluido el Sars-CoV-2) y bacterianas. La hipovitaminosis $D$ es un factor de riesgo identificado para el desarrollo de infecciones por este tipo de microorganismos, razón por la cual la identificación en un momento temprano de los pacientes con deficiencia de vitamina $\mathrm{D}$ y la corrección de sus concentraciones pueden ayudar a prevenir procesos infecciosos pulmonares.

\section{Vitamina D}

Se ha establecido un posible vínculo entre la deficiencia de vitamina $D$ y diversas enfermedades con componente inmunológico e inflamatorio, por ejemplo las infecciones sistémicas ${ }^{1-4}$.

Entre las diversas funciones de la vitamina $D$ figuran la conservación de la salud ósea mediante la regulación de los valores séricos de calcio y fosforo, así como las funciones en la respuesta inmunitaria, y su papel en la proliferación, diferenciación y apoptosis celular. La ingestión de alimentos ricos en vitamina $D$, por ejemplo pescado graso, aceite de hígado de bacalao, yema de huevo y productos lácteos fortificados, es una fuente importante de las reservas corporales; pese a ello, es la producción endógena en la piel a través de la fotosíntesis mediada por los rayos ultravioleta B (UVB) la que genera la mayor cantidad de vitamina circulante y cuyos valores normales giran, según algunas notificaciones, en derredor de $30 \mathrm{ng} / \mathrm{ml}$ (la cifra más aceptada como normal); los valores $<30 \mathrm{ng} / \mathrm{ml}$ se consideran insuficiencia, valores $<20 \mathrm{ng} / \mathrm{ml}$ representan deficiencia y las concentracioes $<10 \mathrm{ng} / \mathrm{ml}$ son ya en adultos osteomalacia y en niños raquitismo'.

La síntesis de la vitamina $D$ derivada de la piel es muy variable, ya que depende de muchos factores, como la pigmentación, la edad, la ropa, el consumo dietético y las condiciones climáticas locales, ya que los valores de 25 -hidroxivitamina $D$ cambian con las estaciones y la exposición a la luz solar. Algunos estudios han demostrado que las cifras séricas de 25-hidroxivitamina $D$ disminuyen en un $20 \%$ desde finales del verano hasta mediados del invierno, mientras que 30 minutos de exposición al sol en todo el cuerpo durante el verano genera vitamina $D$ con rapidez. Otro factor importante en la regulación es el índice de masa corporal (IMC), que se encuentra inversamente relacionado con el valor sérico de la 25-hidroxivitamina $D$ y los pacientes obesos tienen con frecuencia concentraciones subóptimas en el intervalo de 10 a $20 \mathrm{ng} / \mathrm{ml}(25$ a $50 \mathrm{nmol} / \mathrm{L})$; estas diferencias pueden deberse en parte a menores grados de ejercicio y exposición a la luz solar en personas obesas que en sujetos con un índice de masa corporal normal ${ }^{5-9}$.

Para la obtención de metabolitos activos de vitamina D se necesitan dos pasos importantes de hidroxilación, el primero hepático en el cual la 25-hidroxilasa hidroxila a la vitamina $D$ y su resultado es la formación de 25 -hidroxivitamina $\mathrm{D}_{3}\left[25(\mathrm{OH}) \mathrm{D}_{3}\right.$, que es la principal forma circulante y, por lo tanto, útil para determinar el estado de la vitamina D. Con posterioridad, ésta se desplaza unida a las proteínas transportadoras hacia los riñones, donde se somete a un segundo paso de hidroxilación con mediación de la enzima 1 $\alpha$-hidroxilasa CYP27B1 para crear la forma bioactiva $1,25(\mathrm{OH})_{2} \mathrm{D}_{3}{ }^{10}$. Es importante señalar que las enzimas metabolizadoras de la vitamina $D$ se encuentran en otros tejidos, como la placenta, la piel y las células inmunitarias ${ }^{11,12}$.

Las funciones de la vitamina $D$ en su forma activa se ejercen mediante la unión al receptor de vitamina $D$ (RVD), un factor de transcripción regulado por ligando y miembro de la superfamilia de receptores nucleares que se expresa ampliamente en casi todos los tejidos corporales y tipos de células, incluido el sistema inmunitario ${ }^{13}$. Tras la unión del ligando al receptor se forma un complejo RVD- retinoide $X$ con capacidad de unirse a secuencias de DNA específicas en la región promotora de genes denominadas elementos sensibles a la vitamina $\mathrm{D}$, para atraer hacia sí proteínas correguladoras que permiten activar o rechazar la transcripción génica $^{14,15}$. Además, el 1,25(OH $)_{2} \mathrm{D}_{3}$ puede realizar acciones rápidas no genómicas que tienen lugar en segundos a minutos después de la adición de la hormona mediada por una forma unida a la membrana del RVD. $^{16,17}$

La insuficiencia de vitamina $D$ es un problema de salud pública que afecta a miles de personas en todo el mundo y se refleja en alteraciones del sistema 
inmunitario debido a la función inmunomoduladora que ejerce; in vitro, la vitamina $\mathrm{D}$ activa, el calcitriol, estimula la producción de citocinas antiinflamatorias y regula de forma descendente las moléculas activadoras de células inmunitarias proinflamatorias, además de tener efectos positivos sobre la inmunidad innata dado que incrementa la producción de péptidos antivirales en las mucosas ${ }^{18,19}$.

Varias afecciones producen concentraciones séricas muy bajas de 25-hidroxivitamina $D$, entre ellas una ingestión pobre en la dieta y una exposición solar insignificante, mala absorción por enfermedad inflamatoria intestinal, enteropatía por gluten, cirugía gástrica, enfermedad biliar o sobrecrecimiento intestinal; consumo de fármacos anticonvulsivos (p. ej., fenobarbital o fenitoína) y administración de largo plazo de glucocorticoides; además, la deficiencia de vitamina $D$ se ha relacionado con resultados negativos en la salud, como enfermedad ósea, diabetes, hipertensión, enfermedad cardíaca, cáncer y enfermedades autoinmunitarias e infecciosas ${ }^{9,20-25}$.

\section{Vitamina D como inmunomodulador}

El papel inmunomodulador de la vitamina $D$ se ha investigado de forma amplia y gran parte del conocimiento actual procede de estudios que utilizan modelos animales y células humanas in vitro; estos protocolos indicaron que parte de la función inmunitaria innata y adaptativa está regulada por la vitamina D (Lang y Aspinall, 2017). La influencia de la vitamina $D$ en la respuesta inmunitaria humana parece ser dependiente de las concentraciones de $25(\mathrm{OH}) \mathrm{D}_{3}$ y puede conducir a una respuesta aberrante a la infección o incluso a la autoinmunidad en quienes padecen hipovitaminosis ${ }^{26,27}$.

Los mecanismos por los que la vitamina $D$ reduce el riesgo de infección microbiana y la muerte se pueden agrupar en tres categorías: barrera física, inmunidad natural celular e inmunidad adaptativa.

La vitamina $D$ ayuda a mantener las uniones estrechas, las uniones huecas y las uniones adherentes intercelulares mediante la producción de E-cadherina, una molécula de adhesión celular ${ }^{28}$. Varios artículos han revisado de qué modo alteran los virus la integridad de las uniones intercelulares y aumentan la virulencia de virus y bacterias; es en este punto en el que la acción de la vitamina $D$ adopta un papel importante en la prevención de infecciones al conformar parte de la primera barrera de defensa corporal ${ }^{29-31}$.

En segundo lugar, mejora la inmunidad innata a través de la inducción de péptidos antimicrobianos, como la catelicidina humana, LL-3732,33 y diversas defensinas ${ }^{34}$. Las catelicidinas poseen actividades antimicrobianas directas contra bacterias grampositivas y gramnegativas, virus y hongos ${ }^{35}$; estos péptidos derivados del huésped destruyen a los patógenos invasores al modificar sus membranas celulares y pueden, además, neutralizar las actividades biológicas de las endotoxinas ${ }^{36}$. Algunos estudios han mostrado que $1,25(\mathrm{OH})_{2}$ D reduce la replicación viral in vitro e in vivo en cepas de rotavirus ${ }^{37}$. La LL-37 redujo la replicación del virus de la influenza A. Por otro lado, un estudio clínico demostró que la administración de 4,000 UI/día de vitamina $D$ redujo la infección por el virus del dengue ${ }^{38}$. Por otra parte, la vitamina $D$ mejora la inmunidad innata de tipo celular al disminuir la tormenta de citocinas proinflamatorias inducida por el sistema inmunitaria innato en respuesta a infecciones virales y bacterianas, como se observó en pacientes con COVID-19 ${ }^{39}$; con una regulación descendente de la producción de citocinas Th1 proinflamatorias $\gamma$ aumenta la expresión de citocinas antiinflamatorias de los macrófagos ${ }^{27,40}$.

La vitamina $D$ también es un modulador de la inmunidad adaptativa; ya en su forma bioactiva suprime las respuestas mediadas por la célula $T$ colaboradora de tipo 1 (Th1), en particular al suprimir la producción de citocinas inflamatorias IL-2 e interferón $\gamma(\text { INF- } \gamma)^{41}$; asimismo, promueve la producción de citocinas por las células T colaboradoras tipo 2 (Th2), lo que ayuda a mejorar la supresión indirecta de las células Th1 ${ }^{42}$.

La vitamina $D$ promueve reacciones inmunitarias innatas esenciales para la protección temprana contra patógenos y la autofagia, la digestión celular de macromoléculas e inclusiones intracelulares en células inmunitarias parenquimatosas y mononucleares, un proceso vital para la supervivencia celular frente al estrés metabólico y también para eliminar a los microbios intracelulares, como micobacterias, virus y patógenos atípicos y células neoplásicas ${ }^{43}$.

\section{Función de la vitamina D en la enfermedad respiratoria}

En el año 2016, según informe de la OMS, las afecciones respiratorias de origen infeccioso constituían la principal causa de muerte dentro del grupo de enfermedades transmisibles, sobre todo en los países de bajos ingresos; esto representó al año tres millones de defunciones en todo el mundo, con la mayor incidencia en los meses de invierno. Se ha establecido también una prevalencia elevada de hipovitaminosis $D$ con 
Tabla 1. Deficiencia de vitamina $D$ en adultos mayores de países de América más afectados por COVID-19

\begin{tabular}{|c|c|c|c|c|c|c|}
\hline \multirow{2}{*}{$\begin{array}{l}\text { Puesto mundial en } \\
\text { incidencia de COVID-19 }\end{array}$} & \multirow[t]{2}{*}{ País } & \multirow[t]{2}{*}{ n } & \multirow{2}{*}{$\begin{array}{l}\text { Edad (años): } \\
\text { media ( } \pm \text { SD) } \\
\text { o intervalo }\end{array}$} & \multicolumn{2}{|c|}{ Deficiencia de vit. D (\%) } & \multirow[t]{2}{*}{ Referencia y año } \\
\hline & & & & $<25 \mathrm{nmol} / \mathrm{L}$ & $<50 \mathrm{nmol} / \mathrm{L}$ & \\
\hline 1 & Estados Unidos & 4,365 & $76 \pm 5$ & $7(\leq 30)$ & 32 & Eymundsdottir, et al. 2020 (81) \\
\hline 6 & Brasil & 908 & $73 \pm 5$ & 14.4 & $58 \cdot 0$ & Lopes, et al. 2014 (82) \\
\hline 13 & Perú & 204 & $39 \pm 11$ & N/A & 29.4 & Pastor, et al. 2019 (83) \\
\hline 14 & Canadá & 11,306 & $3-79$ & $7.4(<30)$ & 36.8 & Sarafin, et al. 2015 (84) \\
\hline 18 & México & 585 & $41 \pm 15$ & $2 \cdot 0$ & $43 \cdot 6$ & Clark, et al. 2015 (85) \\
\hline 20 & Chile & 686 & $\geq 65$ & $26 \cdot 4(<30)$ & 64.9 & Solis-Urra, et al. 2019 (86) \\
\hline 21 & Ecuador & 2374 & $71 \pm 8$ & N/A & $21 \cdot 6$ & Orces, et al. 2015 (87) \\
\hline
\end{tabular}

variación de acuerdo con las estaciones que coincide con las más bajas concentraciones en los meses de otoño e invierno, lo que ha sugerido una relación entre la vitamina $D$ y las enfermedades respiratorias ${ }^{44}$. En la Tabla 1 se resume el efecto de la deficiencia de vitamina $D$ en adultos mayores de países de América afectados con más frecuencia por COVID-19.

Está bien establecida la función de la vitamina D activada en la respuesta inmunitaria. La presencia del receptor nuclear para vitamina $D$ en células de la inmunidad innata y adaptativa permite una regulación ascendente de la respuesta antiinflamatoria ${ }^{4,34}$; además, los estudios in vitro han demostrado que las células epiteliales respiratorias pueden convertir constitutivamente $25(\mathrm{OH}) \mathrm{D}$ inactivo en $1,25(\mathrm{OH}) \mathrm{D}_{3}$, lo que genera elevadas concentraciones locales de vitamina $D$ activa en el epitelio respiratorio y ello aumenta la expresión de genes regulados por la vitamina $D$ con importantes funciones inmunitarias frente a los diversos lipopéptidos bacterianos y el material genético viral al estimular la respuesta inmunitaria innata y regular la respuesta adaptativa para evitar una cascada inflamatoria excesiva ${ }^{45,46}$. Otro mecanismo que interviene en la actividad de la vitamina $D$ contra las enfermedades respiratorias consiste en su capacidad de inhibir la proliferación del músculo liso de la vía respiratoria y otras vías que participan en su remodelación, como las metaloproteasas de matriz, lo que cobra importancia en enfermedades como cáncer o enfermedad pulmonar obstructiva crónica ${ }^{47}$.

Las infecciones respiratorias de origen viral como la influenza o la infección por el virus sincitial respiratorio (VSR) activan una respuesta inmunitaria que incluye brazos de la inmunidad innata o adaptativa con una infiltración de neutrófilos en la vía respiratoria y un estrechamiento de los bronquiolos ${ }^{48}$. Los estudios aleatorizados han demostrado la mayor incidencia de infecciones respiratorias durante los meses de invierno, un hecho que coincide con una menor exposición solar y valores subóptimos de vitamina $D$, lo que ha servido como base para establecer la hipótesis de la relación entre las concentraciones de esta vitamina y una mayor susceptibilidad a desarrollar infecciones respiratorias ${ }^{20,49,50}$. Durante el curso de una infección que compromete el epitelio respiratorio, las cifras altas de vitamina $D$ pueden suprimir la liberación de citocinas proinflamatorias de los macrófagos, además de aumentar la expresión de péptidos antimicrobianos con actividad antiviral con una producción excesiva de catelicidina como un mecanismo de defensa contra la infección ${ }^{51}$; sin embargo, aunque está bien establecido el papel de la vitamina $D$ como inmunomodulador en las infecciones respiratorias virales, recientes metaanálisis no han logrado demostrar con claridad la función de su complementación en la prevención de dichas infecciones en individuos con hipovitaminosis, por lo que resulta necesario realizar estudios prospectivos aleatorizados que permitan establecer el valor sérico óptimo para prevenir la aparición de las enfermedades de origen viral ${ }^{9,13-16,49,52,53}$.

Por otro lado, en relación con las infecciones respiratorias de origen bacteriano, la acción de la vitamina D contra Mycobacterium tuberculosis se estableció desde la década de 1980, cuando se observó que la adición de vitamina $D$ a células infectadas redujo la carga bacteriana ${ }^{54} \mathrm{y}$ además que el suministro de vitamina D oral, como parte del tratamiento para infecciones micobacterianas, elevó las tasas de éxito ${ }^{55}$. En la actualidad se conoce que el papel de la vitamina $D$ en la enfermedad por Mycobacterium es resultado de 
la muerte de la bacteria mediada por la producción de un péptido antimicrobiano, el LL37, estimulado por la vitamina $D$ en el epitelio bronquial, lo que lleva a una mayor negativización del esputo y mejoría radiológi$\mathrm{ca}^{56}$, además de la reducción del número de bacilos viables en el esputo ${ }^{57}$.

En cuanto a las enfermedades respiratorias inflamatorias de origen no infeccioso, como el asma o la enfermedad pulmonar obstructiva crónica, en las que el proceso fisiopatológico abarca un componente inmunológico con activación de células como macrófagos, neutrófilos o eosinófilos, la actividad de la vitamina $D$ es clara.

La enfermedad pulmonar obstructiva crónica se caracteriza por una obstrucción irreversible al flujo de aire, con un estrechamiento de las vías respiratorias inferiores y un proceso fisiopatológico establecido por estrés oxidativo, inflamación y un desequilibrio entre las proteasas y antiproteasas a nivel pulmonar ${ }^{58,59}$. Algunos estudios han demostrado que las cifras bajas de vitamina $D$ en individuos con EPOC se han vinculado con una mayor frecuencia de exacerbaciones y un curso más grave de la enfermedad ${ }^{60,61}$, además de que existe una relación significativa entre las concentraciones de vitamina $D$ y la función pulmonar dado que las investigaciones muestran que la concentración sérica de $25(\mathrm{OH}) \mathrm{D}$ se vincula con el bajo volumen de $\mathrm{FEV}_{1}$ en pacientes con EPOC ${ }^{62}$.

En relación con el asma, definida como una enfermedad inflamatoria que causa una obstrucción reversible al flujo de aire con síntomas como sibilancias o tos ${ }^{63}$, la acción de la vitamina $D$ puede explicarse por varios mecanismos; en primer lugar, puede disminuir las citocinas inflamatorias como IL-9, IL-5 e IL-13 en células del epitelio respiratorio ${ }^{64} \mathrm{y}$, en segundo lugar, contribuye a superar la resistencia a los glucocorticoides en el tratamiento mediante el aumento de la producción de células $T$ reguladoras secretoras de IL-10 y regular de forma descendente la expresión de FKN, un gen que causa resistencia a los esteroides ${ }^{65}$ al mejorar la respuesta clínica a la enfermedad ${ }^{6}$.

Si se consideran los efectos pleiotrópicos de la vitamina $D$ y su función en la fisiopatología de las enfermedades respiratorias que incluyan un desequilibrio de la respuesta inmunitaria, y sin perder de vista que la causa de la insuficiencia respiratoria en la enfermedad por COVID-19 es la tormenta de citocinas, se ha establecido una relación entre la hipovitaminosis $D$ y un peor curso clínico para los pacientes con insuficiencia respiratoria por COVID-19.

\section{Vitamina D y COVID-19}

Hoy en día, el mundo entero padece la tercera gran pandemia causada por un miembro de la familia Coronavirus, el Sars-Cov2, descrito por primera vez a finales de 2019 en una provincia de Wuhan, China ${ }^{67}$. Poco tiempo después ha afectado a más de 200 países en todo el mundo y causado la muerte de más de $11 \mathrm{mi}-$ llones de personas ${ }^{68}$. La principal causa de mortalidad es el síndrome de dificultad respiratoria aguda posiblemente mediado por una tormenta de citocinas con una respuesta inmunitaria desregulada que incluye a la interleucina 6 , factor de necrosis tumoral $\alpha$, interferón $\gamma$, interleucina $\beta 1$ y otras moléculas de señalización inflamatoria ${ }^{69}$.

Debido al potencial antiinflamatorio bien conocido de la vitamina $D$ y la fisiopatología de la insuficiencia respiratoria observada en la enfermedad por COVID-19, causada por la tormenta de citocinas, se formuló la hipótesis según la cual bajas concentraciones de esta vitamina podrían vincularse con un peor pronóstico y mayor mortalidad de la enfermedad ${ }^{70}$; los aspectos patológicos del COVID-19 suponen una interacción compleja entre el virus y el sistema inmunitario, punto en el cual los valores adecuados de vitamina D modifican la evolución de la enfermedad al reducir la población de macrófagos en el epitelio respiratorio, así como la producción de citocinas y quimiocinas proinflamatorias que empeoran su curso y desenlace clínico ${ }^{71}$; además, existe una indicación de que el mantenimiento de las cifras adecuadas de vitamina D pueden reducir algunas de las secuelas inmunológicas desfavorables tras la infección por COVID-19 secundarias a la elevación retardada de interleucina 6 e interferón $\gamma^{72}$.

Los estudios observacionales retrospectivos realizados en diversos países durante la pandemia por COVID-19 reconocieron la correlación entre las cifras de vitamina $D$ y los casos y la gravedad de COVID-1973-75; un estudio conducido en el sur de Asia demostró una diferencia significativa entre las concentraciones de vitamina $D$ de los casos leves, moderados y graves por enfermedad por COVID-19, con un peor resultado clínico en los individuos con hipovitaminosis $^{73}$. De igual manera, un estudio observacional realizado en Singapur informó que los pacientes con valores adecuados de vitamina $D$ y en quienes se administró un complemento de dicha vitamina, además de vitamina $B_{12}$ y magnesio, requirieron en menor medida oxigenoterapia en comparación con los controles $^{76}$. 
Por otra parte, y según datos epidemiólogos, las tasas de mortalidad por COVID-19 son más altas en los países más alejados del Ecuador, en latitud norte, en comparación con los países cercanos al Ecuador, en latitud sur $^{77}$; esto permitió confirmar la hipótesis de una correlación significativa entre la insuficiencia de vitamina $D$ y las tasas de mortalidad y gravedad por COVID-19 debido a que la luz solar directa en la piel activa la producción endógena de la vitamina $\mathrm{D}$ y en países de latitud norte la intensidad de los rayos UV es más baja que en un país cercano al Ecuador ${ }^{78,79}$. Además, si se considera el confinamiento como una medida importante para la prevención de COVID-19, las horas de exposición a la luz solar han decrecido en todo el mundo y ello produce una mayor prevalencia de hipovitaminosis $D$ y una mayor predisposición a sufrir una enfermedad grave 0 incluso la muerte por COVID-1980.

Otro factor estudiado, y que apoya la hipótesis de la relación entre la vitamina $D$ y la enfermedad por COVID-19, es que las concentraciones séricas de vitamina $\mathrm{D}$ activa tienden a disminuir con la edad ${ }^{5} \mathrm{y}$ son los pacientes de mayor edad los que suelen tener un fenotipo más grave de insuficiencia respiratoria por COVID-19 y una mayor mortalidad concomitante a una prevalencia más elevada de hipovitaminosis $D^{19}$; las razones incluyen menos tiempo al sol y una menor producción de vitamina $\mathrm{D}$ como resultado de cifras más bajas de 7-deshidrocolesterol en la piel, además de cambios en el apetito y la dieta y la polifarmacia ${ }^{12}$; sin valores adecuados de vitamina $D$, los pulmones son vulnerables a la desregulación letal del sistema inmunitario, que incluye tormentas de citocinas, protección insuficiente contra la apoptosis de las células epiteliales y reparación deficiente de las uniones intercelulares destruidas por el virus, entre otros, lo que empeora el desenlace clínico secundario a la enfermedad por COVID-19.

A pesar de la evidencia que demuestra que las cifras bajas de vitamina $D$ se vinculan con una peor evolución de la enfermedad, los estudios clínicos aleatorizados no han logrado demostrar de forma consistente que el complemento de este micronutriente en individuos con infección por COVID-19 mejore el desenlace clínico; en consecuencia, si bien se considera la hipovitaminosis $\mathrm{D}$ como un factor de riesgo adicional para gravedad por COVID-19, aún es necesario realizar más estudios que permitan incluir la vitamina en el tratamiento de pacientes graves; sin embargo, debe considerarse una herramienta de prevención que contribuye a una mejor respuesta inmunitaria en la evolución de la enfermedad.

\section{Conclusión}

Existe una sólida relación entre la hipovitaminosis $D$ y el desarrollo de infecciones del tracto respiratorio inferior, como neumonía, tuberculosis, infección por virus de influenza, e incluso la pandemia actual por COVID-19. Esto podría significar que la identificación temprana de valores bajos de vitamina $D$ y su pronta corrección ayudaría a la prevención del contagio por Sars-CoV-2. Cabe resaltar que, hasta la fecha, no existen registros de deficiencia de vitamina $D$ en pacientes con COVID-19 en Colombia, lo cual hace necesario la realización de estudios clínicos que ayuden a identificar el efecto de este problema en la población.

\section{Conflicto de intereses}

Lo autores declaran no tener ningún conflicto de intereses.

\section{Responsabilidades éticas}

Protección de personas y animales. Los autores declaran que para esta investigación no se han realizado experimentos en seres humanos ni en animales.

Confidencialidad de los datos. Los autores declaran que han seguido los protocolos de su centro de trabajo sobre la publicación de datos de pacientes.

Derecho a la privacidad y consentimiento informado. Los autores declaran que en este artículo no aparecen datos de pacientes.

\section{Bibliografía}

1. Abhimanyu, Coussens AK. The role of UV radiation and vitamin D in the seasonality and outcomes of infectious disease. Photochemical and Photobiological Sciences. 2017. https://doi.org/10.1039/c6pp00355a

2. Agier J, Efenberger M, Brzezi囚ska-Blaszczyk E. Cathelicidin impact on inflammatory cells. In Central European Journal of Immunology. 2015. https://doi.org/10.5114/ceji.2015.51359

3. Alipio M. Vitamin D supplementation could possibly improve clinical outcomes of patients infected with coronavirus-2019 (COVID-2019). SSRN Electronic Journal 2020. https://doi.org/10.2139/ssrn.3571484

4. Arihiro S, Nakashima A, Matsuoka M, Suto S, Uchiyama K, Kato T, et al. Randomized trial of vitamin D supplementation to prevent seasonal influenza and upper respiratory infection in patients with inflammatory bowel disease. Inflammatory Bowel Diseases. 2019. https://doi. org/10.1093/ibd/izy346

5. Banerjee A, Panettieri R. Vitamin D modulates airway smooth muscle function in COPD. In Current Opinion in Pharmacology. 2012 https://doi. org/10.1016/j.coph.2012.01.014

6. Belyaeva IV, Churilov LP, Mikhailova LR, Nikolaev AV, Starshinova AA, Yablonsky PK. Vitamin D, cathelicidin, prolactin, autoantibodies, and cytokines in different forms of pulmonary tuberculosis versus sarcoidosis. Israel Medical Association Journal. 2017.

7. Booth DR, Ding N, Parnell GP, Shahijanian F, Coulter S, Schibeci SD, et al. Cistromic and genetic evidence that the Vitamin $D$ receptor mediates susceptibility to latitude-dependent autoimmune diseases. Genes and Immunity. 2016 https://doi.org/10.1038/gene.2016.12 
8. Bouillon R, Marcocci C, Carmeliet G, Bikle D, White JH, Dawson-Hughes B, et al. Skeletal and extraskeletal actions of vitamin d: current evidence and outstanding questions. Endocrine Reviews. 2019 https://doi. org/10.1210/er.2018-00126

9. Bradshaw MJ, Holick MF, Stankiewicz JM. Vitamin D and multiple sclerosis. In Current Clinical Neurology. 2020. https://doi.org/10.1007/978-3030-24436-1 10

10. Cannell JJ, Vieth R, Umhau JC, Holick MF, Grant WB, Madronich S, et al. Epidemic influenza and vitamin D. In Epidemiology and Infection. 2006 https://doi.org/10.1017/S0950268806007175

11. Cantorna MT, Snyder L, Lin YD, Yang L. Vitamin D and 1,25(OH)2D regulation of T cells. In Nutrients. $2015 \mathrm{https} / / /$ doi.org/10.3390/nu7043011

12. Carlberg C, Campbell MJ. Vitamin D receptor signaling mechanisms: integrated actions of a well-defined transcription factor. In Steroids. 2013 https://doi.org/10.1016/i.steroids.2012.10.019

13. Carmeliet G, Dermauw V, Bouillon R. Vitamin D signaling in calcium and bone homeostasis: a delicate balance. In Best Practice and Research: Clinical Endocrinology and Metabolism. 2015 https://doi.org/10.1016/j. beem.2015.06.001

14. Chambers ES, Nanzer AM, Pfeffer PE, Richards DF, Timms PM, Martineau AR, et al. Distinct endotypes of steroid-resistant asthma characterized by IL-17Ahigh and IFN- $\gamma$ high immunophenotypes: potential benefits of calcitriol. Journal of Allergy and Clinical Immunology. 2015https://doi. org/10.1016/j.jaci.2015.01.026

15. Chen Y, Leng K, Lu Y, Wen L, Qi Y, Gao W, et al. Epidemiological features and time-series analysis of influenza incidence in urban and rural areas of Shenyang, China, 2010-2018. Epidemiology and Infection. $2020 \mathrm{https} / / / \mathrm{doi}$. org/10.1017/S0950268820000151

16. Christakos S, Dhawan P, Verstuyf A, Verlinden L, Carmeliet G. Vitamin D: metabolism, molecular mechanism of action, and pleiotropic effects vitamin D and its bioactivation vitamin D analogs. Physiol Rev. $2018 \mathrm{https} / / /$ doi.org/10.1152/physrev.00014.2015

17. Colotta F, Jansson B, Bonelli F. Modulation of inflammatory and immune responses by vitamin D. In Journal of Autoimmunity. 2017 https://doi. org/10.1016/ijaut.2017.07.007

18. Cui X, Gooch H, Petty A, McGrath JJ, Eyles D. Vitamin D and the brain: Genomic and non-genomic actions. In Molecular and Cellular Endocrinology. 2017 https://doi.org/10.1016/j.mce.2017.05.035

19. Daneshkhah A, Eshein A, Subramanian H, Roy HK, Backman V. The role of vitamin $\mathrm{D}$ in suppressing cytokine storm in COVID-19 patients and associated mortality. MedRxiv. 2020 https://doi.org/10.1101/2020.04.08.20058578

20. de Sá Del Fiol F, Barberato-Filho S, Lopes LC, de Cássia Bergamaschi C. Vitamin $\mathrm{D}$ and respiratory infections. In Journal of Infection in Developing Countries. 2015 https://doi.org/10.3855/idc. 5711

21. De Smet D, De Smet K, Herroelen P, Gryspeerdt S, Martens GA. Vitamin D deficiency as risk factor for severe COVID-19: a convergence of two pandemics. In MedRxiv. 2020 https://doi.org/10.1101/2020.05.01.20079376

22. Nursyam EW, Amin Z, Rumende CM. The effect of vitamin D as supplementary treatment in patients with moderately advanced pulmonary tuberculous lesion. Acta Medica Indonesiana. 2006.

23. El James G, Siddharth N, Rajeev R, Deepak R. Vitamin D level of mild and severe elderly cases of COVID-19: a preliminary report. SSRN (PrePrint). 2020 https://doi.org/10.1101/2020.04.24.20075838V

24. Fabbri M, Paone A, Calore F, Galli R, Gaudio E, Santhanam R, et al. MicroRNAs bind to toll-like receptors to induce prometastatic inflammatory response. Proceedings of the National Academy of Sciences of the United States of America. 2012 https://doi.org/10.1073/ pnas. 1209414109

25. Fischer BM, Pavlisko E, Voynow JA. Pathogenic triad in COPD: oxidative stress, protease-antiprotease imbalance, and inflammation. In International Journal of COPD. 2011 https://doi.org/10.2147/COPD. S10770

26. Fischer K. Vitamin D. In Principles of nutrigenetics and nutrigenomics: fundamentals of individualized nutrition. $2019 \mathrm{https} / / / \mathrm{doi} .0 \mathrm{rg} / 10.1016 /$ B978-0-12-804572-5.00032-X

27. Foong XRE, Bosco A, Troy NM, Gorman S, Hart PH, Kicic A, et al. Identification of genes differentially regulated by vitamin $D$ deficiency that alter lung pathophysiology and inflammation in allergic airways disease. American Journal of Physiology-Lung Cellular and Molecular Physiology. $2016 \mathrm{https}: / /$ doi.org/10.1152/ajplung.00026.2016

28. Ginde AA, Mansbach JM, Camargo CA. Association between serum 25-hydroxyvitamin $D$ level and upper respiratory tract infection in the Third National Health and Nutrition Examination Survey. Archives of Internal Medicine. 2009 https://doi.org/10.1001/archinternmed.2008.560

29. Glass CA, Cash JC, Mullen J. Coronavirus disease 2019 ( $\mathbb{\otimes}$ COVID-19) situation report, 169. Geneva: World Health Organization 2020-07-07, July. https://doi.org/10.1891/9780826153425.0016b

30. Global Initiative for Asthma. Global Initiative for asthma: global strategy for asthma management and prevention (updated 2020). Revue Francaise d'Allergologie et d'Immunologie Clinique. 2020 https://doi.org/10.1016/ S0335-7457(96)80056-6

31. GOLD Assembly. Chronic obstructive pulmonary disease 2019 report global initiative for chronic obstructive lung disease (GOLD). Gold. 2019
32. Gombart AF, Pierre A, Maggini S. A review of micronutrients and the immune system-working in harmony to reduce the risk of infection. Nutrients. 2020. https://doi.org/10.3390/nu12010236

33. Guo C, Rosoha E, Lowry MB, Borregaard N, Gombart AF. Curcumin induces human cathelicidin antimicrobial peptide gene expression through a vitamin $D$ receptor-independent pathway. Journal of Nutritional Biochemistry. 2013. https://doi.org/10.1016/j.jnutbio.2012.04.002

34. Hansdottir S, Monick MM, Hinde SL, Lovan N, Look DC, Hunninghake GW. Respiratory epithelial cells convert inactive vitamin $D$ to its active form: potential effects on host defense. The Journal of Immunology. $2008 \mathrm{ht}-$ tps://doi.org/10.4049/jimmunol.181.10.7090

35. Holick MF. The vitamin D deficiency pandemic: approaches for diagnosis, treatment and prevention. In Reviews in Endocrine and Metabolic Disorders. 2017https://doi.org/10.1007/s11154-017-9424-1

36. Holick MF, Chen TC. Vitamin D deficiency: a worldwide problem with health consequences. American Journal of Clinical Nutrition. 2008 https:// doi.org/10.1093/ajcn/87.4.1080s

37. Huang $C$, Wang $Y, L i X$, Ren $L$, Zhao J, Hu $Y$, et al Clinical features of patients infected with 2019 novel coronavirus in Wuhan, China. The Lancet. 2020 https://doi.org/10.1016/S0140-6736(20)30183-5

38. Ilie PC, Stefanescu S, Smith L. The role of vitamin D in the prevention of coronavirus disease 2019 infection and mortality. Aging Clinical and Experimental Research. $2020 \mathrm{https} / / / \mathrm{doi} .0 \mathrm{org} / 10.1007 / \mathrm{s} 40520-020-01570-8$

39. Infante M, Ricordi C, Sánchez J, Clare-Salzler MJ, Padilla N, Fuenmayor $\mathrm{V}$, et al. Influence of vitamin D on islet autoimmunity and beta-cell function in type 1 diabetes. Nutrients. 2019 https://doi. org/10.3390/nu11092185

40. Jacquillet G, Unwin RJ. (2019). Physiological regulation of phosphate by vitamin D, parathyroid hormone (PTH) and phosphate (Pi). In Pflugers Archiv European Journal of Physiology. 2019 https://doi.org/10.1007/ s00424-018-2231-z

41. Janssens W, Mathieu C, Boonen S, Decramer M. Vitamin D deficiency and chronic obstructive pulmonary disease. A vicious circle. In Vitamins and Hormones. 2011. https://doi.org/10.1016/B978-0-12-386960-9.00017-4

42. Jeffery LE, Burke F, Mura M, Zheng Y, Qureshi OS, Hewison M, et al. 1,25-dihydroxyvitamin D 3 and IL-2 combine to inhibit T cell production of inflammatory cytokines and promote development of regulatory T cells expressing CTLA-4 and FoxP3 . The Journal of Immunology. $2009 \mathrm{https}: / /$ doi.org/10.4049/jimmunol.0803217

43. Jenkinson $C$. The vitamin D metabolome: an update on analysis and function. In Cell Biochemistry and Function. 2019 https://doi.org/10.1002/ cbf. 3421

44. Jones $G$. Expanding role for vitamin $D$ in chronic kidney disease: Importance of blood 25-OH-D levels and extra-renal $1 \alpha-$ Hydroxylase in the Classical and Nonclassical Actions of 1 $\alpha, 25$-Dihydroxyvitamin D3. Seminars in Dialysis. 2007 https://doi.org/10.1111/j.1525-139X.2007.00302.x

45. Kast JI, McFarlane AJ, Głobiðska A, Sokolowska M, Wawrzyniak P, Sanak M, et al. Respiratory syncytial virus infection influences tight junction integrity. Clinical and Experimental Immunology. 2017 https://doi. org/10.1111/cei.13042

46. Keating P, Munim A, Hartmann JX. Effect of vitamin D on T-helper type 9 polarized human memory cells in chronic persistent asthma. Annals of Allergy, Asthma and Immunology. 2014 https://doi.org/10.1016/f. anai.2013.11.015

47. Kochanczyk M, Grabowski F, Lipniacki T. Impact of the contact and exclusion rates on the spread of COVID-19 pandemic. In MedRxiv. 2020 https://doi.org/10.1101/2020.03.13.20035485

48. Laird E, Rhodes J, Kenny RA. Vitamin D and inflammation: potential implications for severity of Covid-19. Irish Medical Journal. 2020

49. Lau FH, Majumder R, Torabi R, Saeg F, Hoffman R, Cirillo JD, et al Vitamin $D$ insufficiency is prevalent in severe COVID-19. MedRxiv. 2020 https://doi.org/10.1101/2020.04.24.20075838

50. Lei GS, Zhang C, Cheng BH, Lee CH. Mechanisms of action of vitamin D as supplemental therapy for pneumocystis pneumonia. Antimicrobial Agents and Chemotherapy. 2017 https://doi.org/10.1128/AAC.01226-17

51. Liu PT, Stenger S, Li H, Wenzel L, Tan BH, Krutzik SR, et al. Toll-like receptor triggering of a vitamin D-mediated human antimicrobial response. Science. 2006https://doi.org/10.1126/science.1123933

52. López-Muñoz P, Beltrán B, Sáez-González E, Alba A, Nos P, Iborra M. Influence of vitamin $D$ deficiency on inflammatory markers and clinical disease activity in IBD patients. Nutrients. 2019 https://doi.org/10.3390/ nu11051059

53. Marino R, Misra M. Extra-skeletal effects of vitamin D. In Nutrients. 2019 https://doi.org/10.3390/nu11071460

54. Martineau AR, James WY, Hooper RL, Barnes NC, Jolliffe DA, Greiller CL, et al. Vitamin D3 supplementation in patients with chronic obstructive pulmonary disease (ViDiCO): a multicentre, double-blind, randomised controlled trial. The Lancet Respiratory Medicine. 2015 https://doi. org/10.1016/S2213-2600(14)70255-3

55. Martineau AR, Jolliffe DA, Hooper RL, Greenberg L, Aloia JF, Bergman $P$, et al. Vitamin $D$ supplementation to prevent acute respiratory tract infections: systematic review and meta-analysis of individual participant data. BMJ (Online). 2017 https://doi.org/10.1136/bmj. 66583 
56. Martínez-Moreno J, Hernández JC, Urcuqui-Inchima S. Effect of high doses of vitamin $\mathrm{D}$ supplementation on dengue virus replication, Toll-like receptor expression, and cytokine profiles on dendritic cells. Molecular and Cellular Biochemistry. 2020 https://doi.org/10.1007/s11010-01903658-w

57. Ng SC, Shi HY, Hamidi N, Underwood FE, Tang W, Benchimol El, et al. Worldwide incidence and prevalence of inflammatory bowel disease in the 21st century: a systematic review of population-based studies. The Lancet. 2017 https://doi.org/10.1016/S01406736(17)32448-0

58. Pham H, Rahman A, Majidi A, Waterhouse M, Neale RE. (2019). Acute respiratory tract infection and 25 -hydroxyvitamin $D$ concentration: a systematic review and meta-analysis. In International Journal of Environmental Research and Public Health. 2019 https://doi.org/10.3390/ijerph16173020

59. Pike JW, Christakos S. Biology and mechanisms of action of the vitamin D hormone. In Endocrinology and Metabolism Clinics of North America. 2017 https://doi.org/10.1016/j.ecl.2017.07.001

60. Pike JW, Meyer MB, Bishop KA. (2012). Regulation of target gene expression by the vitamin $D$ receptor - An update on mechanisms. In Reviews in Endocrine and Metabolic Disorders. 2012 https://doi.org/10.1007/ s11154-011-9198-9

61. Queval CJ, Brosch R, Simeone R. The macrophage: a disputed fortress in the battle against Mycobacterium tuberculosis. In Frontiers in Microbiology. 2017 https://doi.org/10.3389/fmicb.2017.02284

62. Rondanelli M, Miccono A, Lamburghini S, Avanzato I, Riva A, Allegrini P, et al. Self-care for common colds: the pivotal role of vitamin D, vitamin C zinc, and echinacea in three main immune interactive clusters (physical barriers, innate and adaptive immunity) involved during an episode of common colds - practical advice on dosages and on the time to take these nutrients/botanicals in order to prevent or treat common Colds. In Evidence-based Complementary and Alternative Medicine. 2018 https:// doi.org/10.1155/2018/5813095

63. Rossi GA, Fanous H, Colin AA. Viral strategies predisposing to respiratory bacterial superinfections. Pediatric Pulmonology. 2020 https://doi. org/10.1002/ppul.24699

64. Sassi F, Tamone C, D'amelio P. Vitamin D: nutrient, hormone, and immunomodulator. In Nutrients. 2018 https://doi.org/10.3390/ nu10111656

65. Soy M, Keser G, Atagündüz $P$, Tabak F, Atagündüz I, Kayhan S. Cytokine storm in COVID-19: pathogenesis and overview of anti-inflammatory agents used in treatment. In Clinical Rheumatology. 2020 https://doi. org/10.1007/s10067-020-05190-5

66. Tan CW, Ho LP, Kalimuddin S, Cherng BPZ, Teh YE, Thien SY, et al. A cohort study to evaluate the effect of combination vitamin $D$, magnesium and vitamin $\mathrm{B} 12$ (DMB) on progression to severe outcome in older COVID-19 patients. MedRxiv. 2020 https://doi.org/10.1101/2020.06.01.20112334
67. Troeger C, Forouzanfar M, Rao PC, Khalil I, Brown A, Swartz S, et al. (2017). Estimates of the global, regional, and national morbidity, mortality, and aetiologies of lower respiratory tract infections in 195 countries: a systematic analysis for the Global Burden of Disease Study 2015. The Lancet Infectious Diseases. 2017 https://doi.org/10.1016/S1473-3099(17)30396-1

68. Urashima M, Mezawa H, Noya M, Camargo CA. (2014). Effects of vitamin D supplements on influenza A illness during the $2009 \mathrm{H} 1 \mathrm{~N} 1$ pandemic: a randomized controlled trial. Food and Function. $2014 \mathrm{https}: /$ doi.org/10.1039/c4fo00371c

69. Urashima M, Segawa T, Okazaki M, Kurihara M, Wada Y, Ida H. Randomized trial of vitamin $\mathrm{D}$ supplementation to prevent seasonal influenza A in schoolchildren. American Journal of Clinical Nutrition. $2010 \mathrm{https}: / /$ doi.org/10.3945/ajcn.2009.29094

70. Van Reeth K. Cytokines in the pathogenesis of influenza. Veterinary Microbiology. $2000 \mathrm{https} / / /$ doi.org/10.1016/S0378-1135(00)00171-1

71. Vanherwegen AS, Gysemans C, Mathieu C. Regulation of immune function by vitamin $d$ and its use in diseases of immunity. In Endocrinology and Metabolism Clinics of North America. 2017 https://doi.org/10.1016/j. ecl.2017.07.010

72. Wacker M, Holick MF. Sunlight and Vitamin D: a global perspective for health. In Dermato-Endocrinology. 2013 https://doi.org/10.4161/derm.24494

73. Wortsman J, Matsuoka LY, Chen TC, Lu Z, Holick MF. Decreased bioavailability of vitamin $D$ in obesity. American Journal of Clinical Nutrition. $2000 \mathrm{https} / / /$ doi.org/10.1093/ajcn/72.3.690

74. Zavala K, Vazirnia A, Liu PT. Vitamin D and Innate Immunity. In Vitamin D: Fourth Edition. $2017 \mathrm{https}: / /$ doi.org/10.1016/B978-0-12-809963-6.00105-X

75. Zdrenghea MT, Makrinioti H, Bagacean C, Bush A, Johnston SL, Stanciu L. A. Vitamin D modulation of innate immune responses to respiratory viral infections. In Reviews in Medical Virology. 2917 https://doi. org/10.1002/rmv.1909

76. Zendedel A, Gholami M, Anbari K, Ghanadi K, Bachari EC, Azargon A. Effects of vitamin D intake on FEV1 and COPD exacerbation: a randomized clinical trial study. Global Journal of Health Science. 2015 https:// doi.org/10.5539/gjhs.v7n4p243

77. Zhao Y, Ran Z, Jiang Q, Hu N, Yu B, Zhu L, et al. Vitamin D alleviates rotavirus infection through a microrna-155-5p mediated regulation of the TBK1/IRF3 signaling pathway in vivo and in vitro. International Journal of Molecular Sciences. 2019 https://doi.org/10.3390/ijms20143562

78. Zhou YF, Luo BA, Qin LL, Shidoji Y. (2019). The association between Vitamin $D$ deficiency and community-acquired pneumonia: a meta-analysis of observational studies. Medicine (United States). 2019 https://doi. org/10.1097/MD.0000000000017252

79. Zhu N, Zhang D, Wang W, Li X, Yang B, Song J, et al. A novel coronavirus from patients with pneumonia in China, 2019. New England Journal of Medicine. 2020 https://doi.org/10.1056/NEJMoa2001017

80. Zmijewski MA. Vitamin D and human health. In International Journal of Molecular Sciences. 2019 https://doi.org/10.3390/ijms20010145 